\title{
Promoção da saúde e diabetes: discutindo a adesão e a motivação de indivíduos diabéticos participantes de programas de saúde
}

\author{
Health promotion and diabetes: discussing the adherence \\ and motivation of diabetics that participate in health programs
}

Jorge de Assis Costa ${ }^{1}$

Rômulo Sangiorgi Medina Balga ${ }^{1}$

Rita de Cássia Gonçalves Alfenas ${ }^{1}$

Rosângela Minardi Mitre Cotta ${ }^{1}$

${ }^{1}$ Departamento de Nutrição e Saúde, Universidade Federal de Viçosa. Avenida PH Rolfs $s / n^{\circ}$, Campus UFV. 36570-000 Viçosa MG.nyron27@hotmail.com

\begin{abstract}
The objective of the present study was to evaluate, by means of a systematic literature review, the adherence and motivation presented by diabetic patients that participate in health intervention programs, which stimulate the adoption of a healthy life style. The ingestion of an adequate diet and the increase in physical activity, focusing in the disease perception, considering the diabetic patient knowledge, making evident the recommendations that should be followed to reach a better life quality, demonstrating that it is possible to live well even if you have a chronic disease such as diabetes. A literature research in the main public health databases (Medline, Lilacs, Scielo, among others) was conducted from 1994 to 2006. A total of 37 studies focused in the interaction among the subject health and disease versus diabetes were selected. This study mentions some daily difficulties felt by the diabetic patient and his family to control the disease. These difficulties can directly affect the patient adhesion to the prescribed treatment. Therefore, the behavioral and emotional factors presented by each patient must be considered when the health activities are being planned to reach total assistance to this population.
\end{abstract}

Key words Diabetes, Nutritional education, Physical activity, Health promotion, Life style
Resumo Este estudo teve como objetivo analisar, por meio de revisão sistemática, informações publicadas sobre a adesão e a motivação de pessoas portadoras de diabetes mellitus (DM) participantes de programas de saúde que visam ao estímulo à adoção de um estilo de vida saudável. Deu-se ênfase à ingestão de dieta adequada e à realização regular de exercícios físicos, enfocando tanto percepções e conhecimentos acerca da doença pelo portador de DM e seus cuidadores quanto a importância da mudança no estilo de vida, evidenciando-se caminhos a se buscar para melhora da qualidade de vida, demonstrando que é possivel viver bem mesmo sendo portador de uma doença crônica como o DM. Para a revisão sistemática, consultaram-se publicações das principais bases de dados em saúde pública (Medline, Lilacs e Scielo, dentre outras) no período de 1994 a 2006. Foram selecionados 37 artigos que tratam do assunto saúde e doença versus diabetes nas mais variadas formas. Retrataram-se dificuldades diárias sentidas pelo portador de DM e por seus familiares para o controle da doença. Tais dificuldades podem influenciar diretamente na adesão do portador de DM ao tratamento prescrito. Assim, os fatores comportamentais e emocionais apresentados por paciente devem ser considerados no planejamento de ações de saúde para assistência integral a essa população.

Palavras-chave Diabetes, Educação nutricional, Exercício físico, Promoção da saúde, Estilo de vida 


\section{Introdução}

O diabetes mellitus do tipo 2 (DM2) tem alcançado proporções alarmantes, chegando a ser considerado uma epidemia ${ }^{1}$. O número de adultos com diabetes no mundo subirá de 135 milhões, em 1995, para 300 milhões até o ano 2025. Este aumento numérico acontecerá principalmente em países em desenvolvimento. Haverá um aumento de $42 \%$, de 51 milhões a 72 milhões, nos países desenvolvidos, e um aumento de $170 \%$, de 84 milhões a 228 milhões, nos países em desenvolvimento. Assim, antes do ano 2025, 75\% das pessoas com diabetes residirão em países em desenvolvimento, em comparação com o índice de $62 \%$ em $1995^{2}$. A campanha nacional de detecção de diabetes mellitus (DM), realizada no Brasil em 2001, detectou que em 70 milhões de pessoas avaliadas, ou seja, $71 \%$ da população-alvo, foram identificados 3,3 milhões de suspeitos de diabetes ${ }^{3}$. Números da Organização Mundial da Saúde (OMS) estimam que, em todo o globo, 987.000 mortes no ano de 2002 ocorreram por conta do diabetes, representando $1,7 \%$ da mortalidade geral ${ }^{4}$.

Dados recentemente publicados, utilizando outro modelo de relação entre incidência, prevalência e mortalidade específica da doença, indicaram que o excesso de mortalidade global atribuível ao diabetes no ano de 2000 foi estimado em 2,9 milhões de mortes, equivalente a $5,2 \%$ da mortalidade geral, sendo $2-3 \%$ nos países pobres e mais de $8 \%$ em países desenvolvidos, tais como os Estados Unidos e o Canadá ${ }^{\text {. }}$

Esse quadro se tornará cada vez mais grave em razão do aumento no número de doentes. Há uma perspectiva grande no aumento do numero de portadores de diabetes tipo 2 (DM2); a suscetibilidade genética não pode justificar isoladamente esse quadro, sendo indubitavelmente os fatores ambientais parte fundamental desse cenário.

Os hábitos de vida da sociedade moderna, caracterizados pelo elevado consumo de dietas desbalanceadas e reduzida prática de exercícios físicos, têm trazido numerosas implicações para a saúde da população, com aumento da ocorrência de doenças crônicas não transmissíveis, como obesidade, DM, resistência insulínica (RI) e síndrome metabólica ${ }^{6,7}$.

Visando à prevenção da ocorrência de complicações associadas ao DM, órgãos como a Associação Americana de Diabetes ${ }^{7}$ e a Sociedade Brasileira de Diabetes $(\mathrm{SBD})^{8}$ propuseram algumas recomendações, como o uso da contagem de carboidratos, automonitorização da glicemia, uso de tratamento medicamentoso adequado, objetivando maior controle da doença. No entanto, de acordo com a $\mathrm{SBD}^{8}$, a adoção de um estilo de vida adequado, com a prática regular de atividades físicas e a ingestão de dieta adequada, é praticamente duas vezes mais efetiva que o tratamento farmacológico no controle do DM. Na perspectiva tanto do paciente como do profissional da saúde, o tratamento do DM é complexo e difícil de ser realizado, o que tem acarretado dificuldades no controle da doença. Modificações nos hábitos de vida relacionados ao tipo de dieta ingerida, à realização de atividade física, monitorização glicêmica, uso diário de medicamentos e de insulina constituem os fundamentos da terapia ${ }^{8}$.

Neste contexto, o presente artigo analisa, com base em uma revisão de literatura, aspectos relevantes da associação entre mudanças no estilo de vida resultantes da educação, da influência da família, dos amigos, dos cuidadores, do ambiente e do sistema de saúde, visando ao impacto na saúde do portador de DM.

\section{Metodologia}

Realizou-se uma revisão sistemática sobre DM nas publicações das principais bases de dados em saúde pública (Medline, Pubmed, Lilacs e Scielo) durante o período de 1994 a 2006. Foram selecionados 37 artigos que tratam do assunto diabetes e saúde nas mais variadas formas, utilizando-se como palavras-chave os seguintes descritores e suas combinações: diabetes, physical exercise, diet, nutritional education e suas versões em português e espanhol.

\section{Conceito de promoção da saúde e a importância de sua inserção na vida do portador de diabetes mellitus}

Segundo a Carta de Ottawa", a "promoção da saúde" deve ser entendida como a capacitação das pessoas e das comunidades para modificar os determinantes da saúde em benefício da própria qualidade de vida, resultando consequentemente em uma perícia natural no controle deste processo.

O desenvolvimento de doenças crônicas não transmissíveis é complexo, sendo necessárias ações permanentes que não apenas foquem os indivíduos e as famílias de maneira isolada, mas que também levem em consideração os aspectos sociais, econômicos e culturais destes. A baixa aderência aos tratamentos medicamentosos e, 
principalmente, a negligência quanto às mudanças necessárias de estilo de vida fazem com que aproximadamente $50 \%$ dos pacientes portadores de doenças crônicas como o DM não obtenham melhoras no contexto da doença ${ }^{10}$.

Segundo a Organização Pan-Americana da Saúde ${ }^{10}$, o aumento da realização de atividade física e a adoção de hábitos alimentares saudáveis não são apenas um problema individual mas sim um problema social, que exige estratégias de ação voltadas para a população, considerando os aspectos culturais apresentados por ela. É necessário que haja uma transformação na maneira de pensar da população e principalmente na ótica dos técnicos de saúde envolvidos nesse processo. Para que tal fato ocorra, é preciso a promoção de processos participativos, que desenvolvam no indivíduo a capacidade de decisão ante os problemas. A partir da formação do pensamento crítico, o empoderamento da população surge como uma perspectiva de melhoria no quadro da saúde no Brasil, criando um conjunto democratizado com estratégias propostas a partir da promoção da saúde, envolvendo a participação da população, do governo, das instituições públicas e privadas ${ }^{11}$. A extrapolação da saúde para além da prática clínica englobando condições de vida geradas por relações sociais é um importante elemento para se entender o processo saúde-doença ${ }^{12}$. Destacam-se a seguir alguns aspectos relevantes sobre a importância da promoção da saúde na melhora da qualidade de vida do portador de DM, sua relação com seus cuidadores e profissionais da saúde no contexto saúde versus doença.

\section{Ingestão de dieta adequada e prática regular de atividade física, hábitos essenciais na saúde do portador de diabetes}

A prática regular de atividade física é considerada primordial no tratamento do DM. A participação de programas que estimulem a realização de atividade física e o consumo de dieta nutricionalmente adequada, associados à assistência médica, pode reduzir o risco de complicações da doença, além de contribuir para a melhora da qualidade de vida do portador de diabetes ${ }^{8,13,14}$.

Dentre os benefícios da atividade física, temse o aumento da utilização de glicose como combustível para o músculo em atividade, contribuindo para o controle da glicemia ${ }^{15}$. A atividade física regular proporciona um aumento do turnover da insulina, pela maior captação hepática deste hormônio e maior sensibilidade dos recep- tores periféricos ${ }^{16}$. A realização de exercícios físicos, associada à ingestão de dieta adequada, contribui para a melhora no perfil lipídico, diminuindo os riscos de doenças cardiovasculares ${ }^{17}$.

A obtenção do equilíbrio energético e a manutenção do peso corporal adequado, mantidos por meio do consumo de uma dieta balanceada e da prática regular de atividade física, são estratégias importantes na prevenção e tratamento do $\mathrm{DM} 2{ }^{7}$. A dieta indicada para pacientes diabéticos deve conter alto teor de fibra alimentar, já que esta reduz a velocidade de absorção da glicose em nível intestinal, contribuindo para o controle glicêmico e melhoria do perfil lipídico ${ }^{18}$. Além disso, a redução do consumo de gorduras, em especial as saturadas e as trans, em contraposição aos maiores teores de ácidos graxos ômega-3 da dieta, auxiliam na prevenção das complicações vasculares do diabetes ${ }^{19}$, favorecendo a perda de peso e a adequação dos níveis sanguíneos de lipídios ${ }^{20}$. A influência dos micronutrientes no risco de diabetes ainda não foi bem elucidada, embora se proponha que certos micronutrientes afetem diretamente a glicemia e o metabolismo de insulina ${ }^{20}$.

Diferentes estudos mostram que o serviço de saúde não está preparado para orientar corretamente o paciente portador de diabetes quanto aos cuidados a serem tomados quando o assunto é educação nutricional e mudança no estilo de vida $^{21-23}$.

Cotta et al. ${ }^{24}$, em estudo observacional de corte transversal, envolvendo $10,33 \%$ dos hipertensos (150 hipertensos) e 15\% dos diabéticos (30 diabéticos) do município de Teixeiras (MG) identificou-se que os medicamentos $(96,6 \%)$ eram a principal forma de tratamento dessas doenças. Os exercícios físicos eram incorporados ao cotidiano de pequena parte dos entrevistados. Vale ressaltar que grande parcela dos entrevistados era analfabeta $(40,9 \%)$ e a mediana da renda mensal deles era inferior a 0,5 salário mínimo. Foram identificados hábitos alimentares inadequados, representados pelo elevado consumo per capita diário de sal, açúcar e óleo, mostrando assim a necessidade de ações de intervenção por parte do serviço de saúde, voltadas para essa população. Ações efetivas devem ser adotadas de maneira a orientar os indivíduos quanto à prática de hábitos de vida saudáveis, visando modificar os hábitos alimentares errôneos apresentados pela população estudada. Deve-se esclarecer a tais indivíduos que o controle da doença só será efetivo se o tratamento medicamentoso for incorporado em associação à adoção de hábitos de vida saudáveis. 
Boog ${ }^{21}$ pesquisou qual a percepção de médicos e enfermeiros acerca do assunto educação nutricional e como esses profissionais orientavam a mudança do hábito alimentar de seus pacientes. O estudo foi realizado em dois serviços de saúde do município de Campinas (SP). Foram entrevistados nove enfermeiras e oito médicos. Apenas um médico e uma enfermeira afirmaram não ter encontrado dificuldades para abordar questões relativas à nutrição. Nesse sentido, pode-se inferir que a educação nutricional possui valor como ideia, mas há inconsistência quando o assunto envolve responsabilidade profissional.

Sem embargo, Guimarães e Takayanagui ${ }^{23}$ pesquisaram a forma como os pacientes portadores de DM2 recebiam orientações do serviço público de saúde, em uma unidade básica e distrital de saúde da Secretaria Municipal de Ribeirão Preto (SP). Foram entrevistados vinte indivíduos com diagnóstico de DM2, os quais receberam orientações para o tratamento da doença no momento do diagnóstico. Tais orientações foram fornecidas, em sua maioria, por um profissional médico (96,5\%), indicando a ausência da atuação de outras categorias profissionais nesse sentido. Dessas orientações, apenas 17,2\% incluíam as recomendações da Sociedade Brasileira de Diabetes, referentes à dieta a ser ingerida, prática de exercício físico e uso de medicamentos. Por sua vez, $82,8 \%$ dos pacientes foram orientados apenas para o tratamento com dieta e/ ou medicação, sem qualquer instrução para a prática da atividade física.

Assunção et al. ${ }^{22}$ realizaram um estudo transversal, de maio de 1998 a janeiro de 1999, coletando informações relativas ao atendimento dos pacientes diabéticos em 32 postos da rede de atenção primária à saúde da zona urbana de Pelotas (RS), na perspectiva de descrever e avaliar a estrutura de atenção primária em diabetes. Foram entrevistados 378 pacientes e 108 médicos. Cerca de $85 \%$ dos médicos relataram prescrever dieta e $72 \%$, exercício físico como forma de tratamento na consulta inicial. Naquele estudo, $15 \%$ e $28 \%$ dos médicos entrevistados não orientavam seus pacientes quanto à dieta e à prática de exercícios físicos, respectivamente. Todos os médicos relataram solicitar glicemia de jejum e $60 \%$ hemoglobina glicosilada para a monitorização laboratorial dos pacientes. O controle da doença variou de $6 \%$ a $11 \%$, evidenciando baixo controle efetivo do DM. Materiais para dosagem de glicemia, glicosúria e cetonúria foram encontrados em uma minoria deles; apenas três postos faziam a distribuição de insulina diretamente ao pa- ciente, concluindo que a maioria deles apresentava deficiência nos requisitos mínimos necessários à promoção da saúde de pacientes portadores de DM.

Apesar de os estudos evidenciados terem sido realizados em nichos diversos, percebe-se que os resultados são similares. Tais estudos apontam para a necessidade de melhor capacitação dos profissionais de saúde para orientações aos portadores de DM que transcendam o tratamento puramente medicamentoso, e também para o fato de que a ausência de uma equipe multiprofissional leva à vulnerabilidade dos serviços de saúde quanto à adoção dos hábitos de vida saudáveis preconizados pela Sociedade Brasileira de Diabetes e pela Organização Mundial da Saúde. Acentua-se, desta forma, a importância da atuação de uma equipe de saúde multiprofissional como elemento técnico capaz de viabilizar uma melhor qualidade da assistência prestada ao portador de DM.

\section{Percepções acerca da doença: conhecimento dos familiares e do portador de diabetes quanto à importância da mudança do estilo de vida}

As evidências científicas apontam para a importância da mudança no estilo de vida para o paciente portador de diabetes, proporcionando melhor controle metabólico e evitando o aparecimento de complicações causadas pela doença, como complicações macrovasculares e microvasculares como retinopatias, pé diabético, neuropatias, hipertensão arterial ${ }^{25-27}$.

No entanto, a ocorrência de mudanças no estilo de vida para a prevenção de complicações e promoção da saúde no tratamento das doenças crônicas é caracterizada pela baixa adesão pelo portador de diabetes mellitus. Pace et al. ${ }^{28}$ avaliaram o conhecimento dos familiares acerca da problemática do portador de DM em um estudo descritivo realizado no ambulatório de endocrinologia e metabologia do Hospital das Clínicas de Ribeirão Preto, da Universidade de São Paulo (HCFMRP-USP). Foram entrevistados 24 familiares dos pacientes que compareceram aos retornos no período de abril de 2000 a março de 2001. Os familiares relatavam conhecer as causas do DM de forma parcial, sendo que vinte deles informaram melhor sobre o que o diabetes pode causar. Apenas 12 souberam informar corretamente o que é o DM; 17 entrevistados relataram que o paciente segue uma dieta prescrita, no entanto com grande dificuldade de adesão; e ape- 
nas oito praticam atividades físicas regularmente. Apesar de o estudo ter apresentado amostra pequena, os seus resultados ilustram a necessidade das ações educativas no conhecimento dos familiares sobre a doença, pois a família é um fator importante de apoio na adesão do tratamento prescrito pelo portador de $\mathrm{DM}^{29}$.

Não obstante, Peres et al. ${ }^{30}$, em um estudo descritivo exploratório qualitativo, entrevistaram oito mulheres portadoras de DM2, em uma unidade de saúde de Ribeirão Preto (SP). Neste estudo, foram avaliados o conhecimento, o pensamento e os sentimentos de mulheres portadoras de DM com relação à dieta prescrita. Foi evidenciada uma dificuldade no seguimento da dieta prescrita, em razão de fatores como a perda do prazer de comer e beber, da autonomia e da liberdade para se alimentar. Para as entrevistadas, seguir uma dieta restritiva adquire caráter extremamente aversivo, entendendo que ela traz prejuízos à saúde. A ausência de sintomas foi citada como um dos pontos que dificultam o seguimento da dieta. Outras dificuldades citadas por elas foram tocar, olhar e manipular os alimentos durante o seu preparo e não poder ingeri-los. Os alimentos doces despontaram como algo extremamente desejado. Transgressão e desejo alimentar foram relatados igualmente na vida das entrevistadas. Para elas, seguir um padrão dietético recomendado resulta em tristeza, e o ato de comer, muitas vezes, vem acompanhado de medo, culpa e revolta. Vale ressaltar que este último estudo foi realizado com uma população de baixa escolaridade e renda.

Welfer e Leite ${ }^{31}$ realizaram uma pesquisa de natureza qualitativa, exploratória e descritiva por meio de entrevista, envolvendo onze portadores de diabetes, sendo dois do sexo masculino e nove do sexo feminino, com idades entre 42 e 80 anos, residentes no município de Ijuí (RS). O estudo possibilitou identificar que os pacientes portadores de diabetes vivenciavam modificações em seu cotidiano, para a manutenção da estabilidade da doença, especialmente em respeito à alimentação. No entanto, os diabéticos expressaram apresentar certa indignação com relação à dieta prescrita, acumulando certa resistência e não cumprindo o que lhes é recomendado. Outro fato relatado pelos entrevistados foi que às vezes eles alteravam a dosagem da medicação prescrita e ingeriam medicação por conta própria. Além disso, o esquema de rodízio das aplicações de insulina recomendado nem sempre era seguido. A atividade física não era realizada por boa parte dos participantes; a maioria deles desconheciam ser portadores de DM e se descobriram diabéticos quando já apresentavam sinais de complicações, principalmente macro e microvasculares. Porém, os entrevistados relataram gostar de participar das reuniões de grupo, alegando que estas contribuem para o reforço das orientações sobre tratamento e controle da doença.

A descoberta tardia da doença demonstra a importância do diagnóstico precoce para minimizar as complicações causadas pelo DM. Evidenciou-se ainda que, apesar de esses diabéticos fazerem parte de um grupo de apoio, nem sempre eles seguiam as orientações prescritas e ainda transgrediam as orientações medicamentosas, se automedicando, reforçando a necessidade de estabelecimento de estratégias que trabalhem a importância do cuidado e do controle na saúde desses indivíduos. Nesse contexto, vale salientar a importância de trabalho multiprofissional nas diferentes unidades de saúde.

\section{Experiências exitosas no tratamento do diabetes mellitus}

Com o objetivo de analisar o efeito do exercício físico regular no controle glicêmico de diabéticos tipo 2, Silva e Lima ${ }^{32}$ recrutaram indivíduos com idades entre 45 e 75 anos, tratados e não tratados com insulina, na região do Vale do Itajaí (SC). Foram realizados testes de glicemia jejum (GJ), hemoglobina glicada (HbA1) e glicemia capilar dos diabéticos. No total, 33 indivíduos foram submetidos a uma intervenção de exercícios físicos durante dez semanas. Os participantes do estudo foram reavaliados após este período, sendo obtidos os seguintes resultados: glicemia capilar média pré-teste $=179 \mathrm{mg} / \mathrm{dL}$ e pós-teste $=$ $148 \mathrm{mg} / \mathrm{dL}$; HbA1 média pré-teste $=9,5 \%$ e pósteste $=8,5 \%$; GJ média pré-teste $=164,8 \mathrm{mg} / \mathrm{dL}$ e pós-teste $=156,4 \mathrm{mg} / \mathrm{dL}$. De acordo com o Diabetes Control and Complications Trial (DCCT) e UK Prospective Diabetes Study (UKPDS), a redução de 1\% na hemoglobina glicosilada reduz a ocorrência de microangiopatia e neuropatia ${ }^{8}$.

Leite et al..$^{33}$ realizaram um estudo para avaliar o impacto do Staged Diabetes Management (SDM) na melhora do controle glicêmico, perfil lipídico e pressão arterial, e a satisfação dos pacientes com este modelo de atendimento. O SDM é um guia prático, que foi desenvolvido pelo International Diabetes Center (Minneapolis, MN, EUA). Desde 1998, tem sido utilizado como modelo para o treinamento de profissionais da saúde e atendimento aos pacientes no Centro de Dia- 
betes do hospital privado Nossa Senhora das Graças (Curitiba, PR). Tal estudo envolveu a participação de equipe multiprofissional, incluindo endocrinologistas, nutricionistas, enfermeiras e assistente social.

Foram avaliados 78 pacientes que completaram um ano de acompanhamento. Do total de diabéticos envolvidos nesse estudo, 27 eram diabetes do tipo 1, e 51 do tipo 2. Após um ano, a hemoglobina glicosilada diminuiu de $8,06 \% \pm 2,25$ para $7,49 \% \pm 2,0(\mathrm{p}=0,045)$. O perfil lipídico e os níveis pressóricos não apresentaram mudanças significantes. Porém, $80 \%$ dos pacientes estavam satisfeitos com esse atendimento. Dentre algumas das causas para a não aderência ao tratamento prescrito, foram relatadas: residência em outra cidade, o que torna difícil a vinda ao Centro de Diabetes; não continuidade de pagamento do plano de saúde por falta de dinheiro; falta de tempo para ir ao médico; preferência por ser atendido por apenas um médico.

Os resultados mostram que mesmo quando os pacientes são atendidos por uma equipe multiprofissional, que tem como objetivo a abordagem da qualidade de vida deles, $20 \%$ não aderem ao tratamento por motivos diversos, o que nos leva à reflexão de que apenas a mudança do enfoque da equipe de saúde não é suficiente; é necessário encontrar mecanismos negociados entre os usuários dos serviços e a equipe, evitando assim o abandono ao tratamento e agravamento da enfermidade.

Destarte, Zanetti et al.$^{34}$ também avaliaram a implementação do mesmo protocolo Staged Diabetes Management (SDM) no Centro Educativo de Enfermagem para Adultos e Idosos (CEEAI) da Escola de Enfermagem de Ribeirão Preto (EERP-USP). Após um ano de estudo, os autores constataram a existência de um grande desafio para a equipe multiprofissional que acompanhou os pacientes, tanto em relação a sua capacitação em educação em diabetes quanto em relação à compreensão de que a aquisição do conhecimento não se traduz necessariamente em mudança de comportamento.

Os autores também constataram que, além da disponibilização de informações, o paciente diabético precisa ser acompanhado por um longo período de tempo, pois sua evolução não se faz da noite para o dia, e que estar ao seu lado é importantíssimo para que ele possa tomar decisões diante das complicações impostas pela doença. Esse tipo de atendimento evidencia os propósitos da Organização Mundial da Saúde ${ }^{1}$, que visa assegurar que o paciente diabético se perce- ba como regente de sua própria vida, responsabilizando-se pelos cuidados exigidos pela enfermidade. Registrou-se grande satisfação por parte dos usuários, havendo grande adesão ao plano alimentar e à atividade física.

Cazarini et al. ${ }^{35}$, não compreendendo a baixa adesão às atividades educativas realizadas no ambulatório de endocrinologia e metabologia do Hospital das Clínicas da Faculdade de Medicina de Ribeirão Preto, da Universidade de São Paulo (HCFMRP-USP) - que atende uma média de 350 pacientes a cada mês e conta, no entanto, com a participação de apenas vinte pacientes no grupo de educação -, realizaram um estudo descritivo com o objetivo de investigar o motivo pelo qual os pacientes não aderiam ao grupo de educação para diabéticos. Foram entrevistados 66 diabéticos no período de maio a junho de 2000 . Os resultados apontaram que $60,6 \%$ dos investigados sabiam da existência de atividade educativa no hospital, mas apenas $21,2 \%$ participam das atividades educativas oferecidas. Dos que sabiam da existência da atividade educativa, as principais causas mencionadas para a não participação foram: falta de interesse, horário inadequado e dificuldades de transporte. Além disso, os pacientes relataram que não consideravam que as atividades educativas pudessem ser um instrumento capaz de ajudá-los no tratamento da doença e se sentiam desmotivados em participar do grupo. No entanto, os pacientes que participavam do grupo relataram gostar das atividades e informaram que aprendiam muito sobre como lidar com a doença e que os exercícios físicos realizados os ajudavam a diminuir a tensão.

Torres et al ${ }^{36}$ relatam uma experiência de estratégia educativa desenvolvida em ambulatório de especialidade, cujo objetivo é estimular o indivíduo a refletir sobre seu estilo de vida cotidiano relacionado à sua patologia, caracterizando-o como um instrumento de educação em saúde em uma perspectiva de promoção de saúde, prevenção e controle da enfermidade. Para tal, foi implementada uma dinâmica que teve por base o uso de jogos educativos realizados em grupos. As técnicas pedagógicas utilizadas para a sistematização da dinâmica foram: curso de orientação em DM, consulta individual, grupo operativo e uso de material educativo de comunicação e aprendizagem em forma de jogo. Participaram do estudo 178 indivíduos; aproximadamente 148 destes tinham idades entre 51 e 70 anos, $73 \%$ e $70 \%$ eram do sexo feminino e masculino, respectivamente, sendo que $57 \%$ dos participantes informaram não saber ler nem escrever. Apesar do grande número 
de pessoas analfabetas, a dinâmica de grupo resultou em estimulação da relação entre os participantes e os profissionais de saúde, facilitando o diálogo entre as pessoas com os mesmos objetivos, possibilitando troca de informações. Os participantes compartilharam suas experiências comuns, que auxiliaram no entendimento da doença, permitindo-lhes expressar dúvidas e expectativas e possibilitando o apoio mútuo.

Maia e Araújo ${ }^{37}$ relataram outro tipo de vivência no que se refere à educação em diabetes, na forma de colônia de fim de semana, oferecido pelo projeto educacional Diabetes Weekend (DW). O objetivo do referido projeto é informar e alertar profissionais de saúde a respeito da importância de se prevenirem as complicações do diabetes mellitus do tipo 1 (DM1). Por meio deste projeto, portadores de DM são educados em relação aos riscos de complicações associadas à doença e orientados quanto às novas formas de terapia do DM1. No DW, a sala de aula é a natureza, e as lições são repassadas de maneira informal. A colônia ocorre sempre em sítios, fazendas e cidades históricas, próximas a Belo Horizonte (MG). No mínimo $20 \%$ das vagas são preenchidas por participantes de nível socioeconômico baixo, participando cerca de quarenta a cinquenta pessoas por vez. Nos resultados obtidos, observa-se a ocorrência de maior conscientização. Dentre as varias faixas etárias que participaram do projeto, a maior aceitação com relação à doença foi dos adolescentes, após participação na colônia de fim de semana.

\section{Considerações finais}

Os eixos de discussão do referido estudo apontam as dificuldades apresentadas pelos portadores de DM e por seus cuidadores. A obtenção de um bom controle metabólico está em geral intimamente relacionada à ingestão de dieta adequa$\mathrm{da}$, à realização regular de atividade física e ao seguimento da terapêutica medicamentosa prescrita. A falta de conhecimento acerca da doença tanto dos cuidadores quantos dos próprios pacientes, associada à inadequada capacitação e integração entre os profissionais de saúde, relaciona-se diretamente ao problema da adesão. Essas constatações apontam para a ineficácia das estratégias tradicionais, sendo necessário que se incorporem nos serviços de saúde novas abordagens capazes de motivar os portadores de DM. Vale ressaltar a importância de se sensibilizar o paciente portador de DM para a adoção de novos hábitos e estilo de vida, conscientizando-os sobre os riscos a que estão submetidos.

O apoio da família e dos amigos (rede de apoio) é primordial para a conscientização das mudanças necessárias ao sucesso do tratamento. O portador de DM deve estar sensibilizado sobre a importância de se promover saúde para melhorar sua vida cotidiana. Nesse caso, a promoção de saúde não se refere à busca de subsídios para que a doença não se estabeleça, uma vez que no portador de DM ela já se instalou; o enfoque, neste caso, refere-se à importância de que o diabético se conscientize que é possível levar uma vida saudável e normal quando se é portador de uma doença crônica. Nesse sentido, a mudança nos hábitos de vida é de fundamental importância, não só para o diabético mas também para aqueles que estão ao seu redor, evitando assim que indivíduos predispostos ao diabetes desenvolvam também a doença.

A atuação da equipe multidisciplinar no cuidado à saúde do paciente é de extrema importância. No entanto, antes de se iniciar a orientação específica sobre o diabetes, é fundamental que toda a equipe conheça os padrões individuais de resposta do paciente em relação aos seus sentimentos, angústias, ansiedades, conflitos e necessidades, estabelecendo um vínculo afetivo para, posteriormente, em conjunto, traçar estratégias, a serem alcançadas a curto, médio e longo prazos, que visem ao controle metabólico do paciente.

Por fim, vale salientar que no presente estudo foram retratadas algumas das dificuldades diárias sentidas pelo portador de DM e pelos seus familiares para o controle da doença. Essas dificuldades podem influenciar na adesão do portador de DM ao tratamento prescrito. Assim, os fatores comportamentais e emocionais apresentados por cada diabético devem ser considerados no planejamento de ações de saúde voltadas para a assistência integral a essa população. No entanto, ainda é necessária a condução de estudos visando identificar aspectos pedagógicos eficazes no aumento da motivação e da adesão dos portadores de DM aos programas de intervenção, compatibilizando, desta forma, conhecimento e práticas pessoais promotoras da saúde. 


\section{Colaboradores}

JA Costa e RSM Balga participaram do planejamento, revisão bibliográfica e redação do artigo; RCG Alfenas participou da orientação, do acompanhamento do trabalho e revisão do artigo; RMM Cotta, da idealização, orientação, correção, acompanhamento do trabalho e revisão.

\section{Referências}

1. World Health Organization. Diet, nutrition and the prevention of chronic diseases. Report WHO Consultation. Geneva: WHO; 2003. (WHO Technical Report Series, 916).

2. King H, Aubert RE, Herman WH. Global burden of diabetes, 1995-2025. Diabetes Care 1998; 21:14141431.

3. Barbosa RB, Barceló A, Machado CA. Campanha Nacional de Deteç̧ão de Casos Suspeitos de diabetes mellitus no Brasil: relatório preliminar. Rev Panam Salud Publica/Pan Am J Public Health 2001; 10(5):328-333.

4. World Health Organization. The World Health Report 2003. Geneva: WHO; 2003.

5. Roglic G, Unwin N, Bennett PH, Mathers C, Tuomilehto J, Nag S, Conolly V, King H. The burden of mortality attributable to diabetes: realistic estimates for the year 2000. Diabetes Care 2005; 28(9):21302135.

6. Grundy SM. Obesity, metabolic syndrome, and coronary atherosclerosis. Circulation 2002; 105:26962698.

7. American Diabetes Association. The prevention or delay of type 2 diabetes (Position Statement). Diabetes Care 2004; 27(Suppl.1):S47-S54.

8. Sociedade Brasileira de Diabetes. Atualização brasileira sobre diabetes. Rio de Janeiro: Diagraphic; 2006.

9. Brasil. Ministério da Saúde. Secretaria de Políticas de Saúde. Projeto Promoção da Saúde. As cartas da Promoção da Saúde. Brasília: Ministério da Saúde; 2002.

10. Organização Pan-Americana da Saúde. Doenças crônico-degenerativas e obesidade: estratégia mundial sobre alimentação saudável, atividade física e saúde. [site da Internet]. [acessado 2007 jun 25]. Disponível em: http://www.opas.org.br/sistema/arquivos/d_cronic.pdf

11. Bydlowski CR, Westphal MF, Pereira IMTB. Promoção da saúde: porque sim e porque não! Saúde e Sociedade 2004; 13(1):14-24.

12. Marcondes WB. A convergência de referências na promoção da saúde. Saúde e Sociedade 2004; 13(1):513.

13. Mercury N, Assad D. Atividade física e diabetes mellitus. Diabetes Clínica 2001; 4:347-349.

14. Molena-Fernandes CA, Junior NN, Tasca RS, Pelloso SM, Cuman RKN. A importância da associação de dieta e de atividade física na prevenção e controle do diabetes mellitus tipo 2. Acta Sci Health Sci 2005; 27(2):195-205.

15. Mercuri N, Arrechea V. Atividade física e diabetes mellitus. Diabetes Clínica 2001; 5:347-349. 
16. Eriksson J, Teimela S, Koivisto VA. Exercise and the metabolic syndrome. Diabetologia 1997; 40:125-135.

17. Thompson FE, Byers T. Dietary assessment manual. J Nutr 1998; 124:22-26.

18. Würsch P, Pi-Sunyer EX. The role of viscous soluble fiber in the metabolic control of diabetes. Diabetes Care 1997; 20:1774-1789.

19. Hu FB, Manson JE, Stampfer MJ, Colditz G, Liu S, Solomon CG, Willett WC. Diet, lifestyle, and the risk of type 2 diabetes mellitus in women. $N$ Engl J Med 2001; 345:790-797.

20. Franz MJ, Bantle JP, Beebe CA, Brunzell JD, Chiasson JL, Garg, A, Holzmeister LA, Hoogwerf B, Mayer-Davis E, Mooradian A, Purnell JQ, Wheeler M. Evidence-based nutrition principles and recommendations for the treatment and prevention of diabetes and related complications. Diabetes Care 2002; 25:148-198.

21. Boog MCF. Educação nutricional em serviço públicos de saúde. Cad Saude Publica 1999; 15(Supl. 2): 139-147.

22. Assunção MCF, Santos IS, Gigante DP. Atenção primária em diabetes no Sul do Brasil: estrutura, processo e resultado. Rev Saude Publ 2001; 35(1):8895.

23. Guimarães FPM, Takayanagui AMM. Orientações recebidas do serviço de saúde por pacientes para o tratamento do portador de diabetes mellitus tipo 2 . Rev Nutr 2002; 15(1):37-44.

24. Cotta RMM, Batista KCS, Reis RS, Souza GA, Dias G, Castro FAF, Alfenas RCG. Perfil sociossanitário e estilo de vida de hipertensos e/ou diabéticos, usuários do Programa de Saúde da Família - município de Teixeiras, MG. Cien Saude Colet 2009; 14(4):1251-1260.

25. Gross JL, Nehme M. Detecção e tratamento das complicações crônicas do diabetes melito: Consenso da Sociedade Brasileira de Diabetes e Conselho Brasileiro de Oftalmologia. Rev Ass Med Brasil 1999; 45(3):279-284.

26. Assis MAA, Nahas MV. Aspectos motivacionais em programas de mudança de comportamento alimentar. Rev Nutr 1999; 12(1):33-41.

27. Sartorelli DS, Franco LJ. Tendências do diabetes mellitus no Brasil: o papel da transição nutricional. Cad Saude Publ 2003; 19(Supl.1):S29-S36.

28. Pace AE, Nunes PD, Ochoa-Vigo K. O conhecimento dos familiares acerca da problemática do portador de diabetes mellitus. Rev Latino-Am Enferm 2003; 11(3):312-319.

29. Wang C, Fenske MM. Self-care of the adults with non-insulindependent diabetes mellitus: influence of family and friends. Diabetes Educ 1996; 22(5):465470 .
30. Peres DS, Franco LJ, Santos MA. Comportamento alimentar em mulheres portadoras de diabetes tipo 2. Rev Saude Publ 2006; 40(2):310-317.

31. Welfer M, Leite MT. Ser portador de diabetes tipo 2: cuidando-se para continuar vivendo. Scientia Medica 2005; 15(3):148-155.

32. Silva CA, Lima WC. Efeito benéfico do exercício físico no controle metabólico do diabetes mellitus tipo 2 a curto prazo. Arq Bras Endocrinol Metab 2002; 46(5):550-556.

33. Leite SAO, Costa PABC, Guse C, Dorociaki JG, Silveira MC, Teodorovicz R, Martinatto JS, Niclewicz EA. Enfoque multidisciplinar ao paciente diabético: avaliação do impacto do Staged Diabetes Management em um sistema de saúde privado. Arq Bras Endocrinol Metab 2001; 45(5):481-486.

34. Zanetti ML, Otero LM, Freitas MCF, Santos MA, Guimarães FPM, Couri CEB, Peres D, Ferronato AA, Sasso KD, Barbieri AS. Atendimento ao paciente diabético utilizando o protocolo Staged Diabetes Management: relato de experiência. RBPS 2006; 19(4):253-260.

35. Cazarini RP, Zanetti ML, Ribeiro KP, Pace AE, Foss MC. Adesão a um grupo educativo de pessoas portadoras de diabetes mellitus: porcentagem e causas. Medicina 2002; 35:142-150.

36. Torres HC, Hortale VA, Schall V. A experiência de jogos em grupos operativos na educação em saúde para diabéticos. Cad Saude Publica 2003; 19(4):10391047.

37. Maia FFR, Araújo LR. Projeto Diabetes Weekend: proposta de educação em diabetes mellitus tipo 1 . Arq Bras Endocrinol Metab 2002; 46(5):566-573.

Artigo apresentado em 04/10/2007

Aprovado em 27/06/2008

Versão final apresentada em 10/07/2008 